\title{
MicroRNA-873 inhibits the progression of thyroid cancer by directly targeting ZEB1
}

\author{
DAN JIAO $^{1}$, FENG GUO ${ }^{1}$ and QINGFENG FU ${ }^{2}$ \\ ${ }^{1}$ Department of Ultrasound; ${ }^{2}$ Jilin Provincial Key Laboratory of Surgical Translational Medicine, \\ Division of Thyroid Surgery, China-Japan Union Hospital of Jilin University, Changchun, Jilin 130033, P.R. China
}

Received March 30, 2018; Accepted May 1, 2019

DOI: $10.3892 / \mathrm{mmr} .2019 .10381$

\begin{abstract}
Recent evidence has revealed that certain microRNAs (miRNAs) are dysregulated in thyroid cancer (TC), which has been associated with the pathogenesis and development of TC. Therefore, improved understanding of the detailed roles of miRNAs that are aberrantly expressed in TC may aid the development of valuable therapeutic methods for the management of patients with this malignancy. In this study, miRNA-873-5p (miR-873) expression in TC tissues and cell lines was detected by reverse-transcription quantitative polymerase chain reaction (RT-qPCR). MTT and cell invasion assays were performed to investigate the effects of miR-873 overexpression on TC cell proliferation and invasion in vitro. Bioinformatics analysis, luciferase reporter assay, RT-qPCR and western blot analysis were employed to evaluate whether zinc finger E-box-binding homeobox 1 (ZEB1) is a direct target of miR-873 in TC cells. The present study reported that miR-873 was weakly expressed in human TC tissues and cell lines. Functionally, miR-873 overexpression suppressed TC cell proliferation and invasion in vitro. Mechanistically, ZEB1 was predicted to be a putative target of miR-873. In addition, miR- 873 was proposed to directly bind to the 3'-untranslated region of ZEB1 and decrease its expression in TC cells at the mRNA and protein levels. Furthermore, ZEB1 expression was significantly upregulated in TC tissues and negatively correlated with miR-873 expression. Furthermore, ZEB1 restoration partially reversed the suppressive effects of miR-873 overexpression on TC cell proliferation and invasion. These results demonstrated that miR-873 may serve tumour-suppressive roles in the progression of TC, and its suppressive effects could be mediated by the inhibition of
\end{abstract}

Correspondence to: Professor Qingfeng Fu, Jilin Provincial Key Laboratory of Surgical Translational Medicine, Division of Thyroid Surgery, China-Japan Union Hospital of Jilin University, 126 Xiantai Road, Changchun, Jilin 130033, P.R. China

E-mail: huaxia_service@126.com

Key words: thyroid cancer, microRNA-873, zinc finger E-box-binding homeobox 1, proliferation, invasion
ZEB1. Therefore, miR-873 may be a valuable therapeutic target in the management of patients with TC.

\section{Introduction}

Thyroid cancer (TC) is one of the most common types of endocrine tumour with a rapidly increasing morbidity rate in the last several decades (1). Based on its histology, TC can be divided into four subtypes, including papillary, follicular, medullary and anaplastic TCs (2). Papillary TC is the most common subtype of thyroid cancer and accounts for $~ 85-90 \%$ of all TC cases (3). Surgical resection together with radioiodine and thyroid-stimulating hormone inhibition therapy remains as the main treatment for patients with TC (4). The majority of TC patients exhibit satisfactory treatment outcomes; however, the prognosis of TC patients with local recurrence or distant metastasis is markedly poor $(5,6)$. Unfortunately, the mechanisms underlying the relapse and metastasis of TC remain largely unknown. Therefore, understanding of the mechanisms of the initiation and progression of TC may aid the development of effective therapeutic strategies to improve the prognosis of TC patients at an advanced stage.

MicroRNAs (miRNAs/miRs) are an abundant class of 18-25 nucleotide-long, single-stranded RNA molecules that serve a critical role in tumourigenesis and tumour development (7). These molecules can modulate gene expression by promoting mRNA cleavage and/or inducing translational suppression by completely or partially complementary binding to the 3'-untranslated regions (3'-UTRs) of their target genes (8). At present, $>2,000$ miRNAs have been identified in the human genome, and these miRNAs have been proposed to regulate the expression of $>30 \%$ of all protein-coding genes (9). An increasing number of studies have reported that the expression of miRNAs is altered in almost all types of human malignancy, such as TC (10), hepatocellular carcinoma (11), breast cancer (12) and gastric cancer (13). The dysregulation of miRNAs is involved in the oncogenesis of TC, and regulates numerous pathological behaviours, such as cellular growth, cycle, apoptosis, metastasis and epithelial-mesenchymal transition (14-16). Considering their important roles in TC, miRNAs may be considered as potential novel diagnostic and therapeutic biomarkers for treating patients with this disease.

miR-873-5p (miR-873) is dysregulated and serves crucial roles in the pathogenesis and progression of various human 
cancer types (17-20); however, the expression, roles and molecular mechanism of miR-873 in TC remain unknown. Therefore, the present study aimed to determine the expression profile of miR-873 in TC tissues and cell lines, examine the roles of miR-873 in TC progression and determine the mechanism underlying the action of miR-873 in TC cells. The present study revealed that miR-873 is downregulated in TC, and inhibits TC cell proliferation and invasion by directly targeting zinc finger E-box-binding homeobox 1 (ZEB1), suggesting that this miRNA may be a potential therapeutic agent for the treatment of patients with this malignancy.

\section{Materials and methods}

Patients and tissue samples. In total, 35 pairs of tissue samples, each comprising TC and adjacent normal tissues, were obtained from patients (16 males, 19 females; age range, 28-63 years) who received surgery at the China-Japan Union Hospital of Jilin University between May 2016 and April 2017. All 35 cases were pathologically diagnosed as TC. The TC tissue samples were classified according to the World Health Organization standards (21). Of note, 24 of the 35 cases were classified as low-grade (T1 and T2), and 11 cases were classified as high-grade (T3 and T4); 9 of the 35 TC patients were diagnosed with lymph node metastasis. After resection, all tissue specimens were immediately frozen in liquid nitrogen and then stored at $-80^{\circ} \mathrm{C}$. None of the TC patients had been treated with any preoperative treatment, including chemotherapy, radiotherapy or thyroid-stimulating hormone inhibition therapy before surgery. The present study was approved by the medical ethics committee of the China-Japan Union Hospital of Jilin University. In addition, written consent was obtained from all participants.

Cell lines and culture conditions. One normal human thyroid cell line (HT-ori3) and two human TC cell lines (TPC-1 and HTH83) were purchased from the American Type Culture Collection (Manassas, VA, USA). All cell lines were cultured in Dulbecco's Modified Eagle's medium (DMEM) containing $10 \%$ fetal bovine serum (FBS) and $1 \%$ penicillin/streptomycin mixture (all Gibco; Thermo Fisher Scientific, Inc., Waltham, MA, USA), and grown at $37^{\circ} \mathrm{C}$ in a $5 \% \mathrm{CO}_{2}$ humidified incubator.

Transient transfection. miR-873 mimics and corresponding negative control mimics (miR-NC) were purchased from Shanghai GenePharma, Co., Ltd. (Shanghai, China). The miR-873 mimics sequence was 5'-GCAGGAACUUGUGAG UCUCCU-3' and the miR-NC sequence was 5'-UUCUCC GAACGUGUCACGUTT-3'. ZEB1-overexpressing plasmid pcDNA3.1-ZEB1 and empty plasmid pcDNA3.1 were chemically produced by Chinese Academy of Sciences (Changchun, China). These miRNA mimics $(100 \mathrm{pmol})$ and plasmids $(4 \mu \mathrm{g})$ were transfected into cells using Lipofectamine ${ }^{\circledR}$ 2000 (Invitrogen; Thermo Fisher Scientific, Inc.), according to the manufacturer's protocols. Cotransfection of miRNA mimics (100 pmol) and plasmid $(2 \mu \mathrm{g})$ were performed using Lipofectamine ${ }^{\circledR} 2000$ (Invitrogen; Thermo Fisher Scientific, Inc.). Reverse transcription-quantitative PCR (RT-qPCR) analysis and MTT assay were performed at $48 \mathrm{~h}$ and $24 \mathrm{~h}$ post-transfection. Cell invasion assay was performed $48 \mathrm{~h}$ after transfection. Cells were collected $72 \mathrm{~h}$ after transfection to perform western blot analysis.

$R T-q P C R$ analysis. Total RNA of the tissue samples or cells was isolated using TRIzol ${ }^{\circledR}$ (Thermo Fisher Scientific, Inc.) in accordance with the manufacturer's protocol. For the detection of miR-873 expression, total RNA was reverse transcribed into complementary DNA (cDNA) using the TaqMan MicroRNA Reverse Transcription Kit (Applied Biosystems; Thermo Fisher Scientific, Inc.) according to the manufacturer's protocol. The expression levels of miR-873 were determined using the TaqMan MicroRNA PCR kit (Applied Biosystems; Thermo Fisher Scientific, Inc.). The thermocycling conditions were as follows: One cycle at $50^{\circ} \mathrm{C}$ for $2 \mathrm{~min}$ and at $95^{\circ} \mathrm{C}$ for $10 \mathrm{~min}$, followed by 40 cycles of denaturation at $95^{\circ} \mathrm{C}$ for $15 \mathrm{sec}$, and annealing/extension at $60^{\circ} \mathrm{C}$ for $60 \mathrm{sec}$. For the quantification of ZEB1 mRNA expression levels, cDNA was synthesized from total RNA using the PrimeScript RT Reagent Kit (Takara Biotechnology Co., Ltd., Dalian, China). ZEB1 mRNA expression was assessed by PCR amplification using the SYBR Premix Ex Taq ${ }^{\mathrm{TM}}$ Kit (Takara Biotechnology Co., Ltd.). The thermocycling conditions were as follows: Initial denaturation at $95^{\circ} \mathrm{C}$ for $5 \mathrm{~min}$, followed by 40 cycles of $95^{\circ} \mathrm{C}$ for $30 \mathrm{sec}$ and $65^{\circ} \mathrm{C}$ for $45 \mathrm{sec}$. All reaction was performed using the Applied Biosystems 7500 real-time PCR system (Thermo Fisher Scientific, Inc.) and repeated three times. U6 small nuclear RNA and GAPDH were used for the normalization of miR-873 and ZEB1 mRNA expression, respectively. Relative gene expression was analysed using the $2^{-\Delta \Delta \mathrm{Cq}}$ method (22). The primers were designed as follows: miR-873 forward, 5'-GCA GGAACUUGUGAGUCUCCU-3' and reverse, 5'-AGGAGA CUCACAAGUUCCUGC-3'; U6 forward, 5'-TTCACGAAT TTGCGTGTCAT-3' and reverse, 5'-CGCTTCACGAATTTG CGTGTCAT-3'; ZEB1 forward, 5'-AAGTGGCGGTAGATG GTA-3' and reverse, 5'-TTGTAGCGACTGGATTTT-3'; and GAPDH forward, 5'-CCCCTTCATTGACCTCAACT-3' and reverse, 5'-ATGAGTCCTTCCACGATACC-3'.

MTT assay. At $24 \mathrm{~h}$ following transfection, cells were seeded into 96-well plates at a concentration of $3 \times 10^{3}$ cells/well. Analysis of cell proliferation was performed using an MTT assay at four time points: $0,24,48$ and $72 \mathrm{~h}$ after inoculation. In total, $20 \mu \mathrm{l}$ of MTT solution ( $5 \mathrm{mg} / \mathrm{ml}$; Sigma-Aldrich; Merck KGaA, Darmstadt, Germany) was added into each well and incubated at $37^{\circ} \mathrm{C}$ under $5 \% \mathrm{CO}_{2}$ for another $4 \mathrm{~h}$. The culture medium was discarded, and $100 \mu \mathrm{l}$ of dimethyl sulfoxide (Sigma-Aldrich; Merck KGaA) was added into each well to dissolve the formazan precipitates. Finally, the absorbance was detected at a wavelength of $490 \mathrm{~nm}$ using a microplate reader (BioTek Instruments, Inc., Winooski, VT, USA).

Cell invasion assay. Transwell chambers ( $8-\mu \mathrm{m}$ pore size) coated with Matrigel (BD Biosciences, Franklin Lakes, NY, USA) were used to investigate cell invasion. Transfected cells were harvested, suspended in FBS-free DMEM and seeded at a concentration of $1 \times 10^{5}$ cells/well into the upper chambers. The lower chambers were coated with $500 \mu 1$ of DMEM containing 20\% FBS (Gibco; Thermo Fisher Scientific, Inc.) to serve as a chemo-attractant. The chambers were incubated 
at $37^{\circ} \mathrm{C}$ in a $5 \% \mathrm{CO}_{2}$ humidified incubator for $24 \mathrm{~h}$. At the end of the incubation, non-invaded cells were gently removed using a cotton swab. The invaded cells were fixed with $100 \%$ methanol at room temperature for $20 \mathrm{~min}$ and stained with $0.1 \%$ crystal violet at room temperature for $20 \mathrm{~min}$ (both from Beyotime Institute of Biotechnology, Haimen, China). The invasive ability of cells was determined by counting the number of invaded cells under an inverted light microscope (IX83; magnification, x200; Olympus Corporation, Tokyo, Japan) in five independent visual fields each chamber.

Bioinformatics analysis. TargetScan (version 7.2; release, March 2018; http://www.targetscan.org/vert_72/) and microRNA (release, August 2010; http://www.microrna.org/ microrna/home.do) were used to predict the putative targets of miR-873.

Luciferase reporter assay. The wild-type (Wt) and mutant (Mut) 3'-UTRs of ZEB1 were chemically synthesised by Shanghai GenePharma Co., Ltd., and cloned into the pmirGLO luciferase reporter vector (Promega Corporation, Madison, WI, USA), to form the pmirGLO-ZEB1-3'-UTR Wt and pmirGLO-ZEB1-3'-UTR Mut vectors, respectively. Cells were inoculated into 24 -well plates with an initial density of $1 \times 10^{5}$ cells/well, and the cells were co-transfected with pmirGLO-ZEB1-3'-UTR Wt or pmirGLO-ZEB1-3'-UTR Mut, and miR-NC or miR-873 using Lipofectamine 2000, in accordance with the manufacturer's instructions. After $48 \mathrm{~h}$ post-transfection, cells were assayed using the Dual-Luciferase ${ }^{\circledR}$ Reporter Assay System (Promega Corporation). Firefly luciferase activity was normalized to that of Renilla.

Protein extraction and western blot analysis. Cells or tissue samples were lysed in radioimmunoprecipitation assay lysis and extraction buffer (Thermo Fisher Scientific, Inc.) for $30 \mathrm{~min}$ on ice to obtain protein. The concentration of total protein was determined using a BCA Protein Assay Kit (Beyotime Institute of Biotechnology). Equal amounts of protein $(30 \mu \mathrm{g})$ was separated by $10 \%$ SDS-PAGE and transferred onto polyvinylidene fluoride membranes (EMD Millipore, Billerica, MA, USA) followed by blocking in 5\% nonfat milk diluted at room temperature for $2 \mathrm{~h}$ in Tris-buffered saline containing $0.1 \%$ Tween-20 (TBST). Afterwards, the membranes were incubated overnight at $4{ }^{\circ} \mathrm{C}$ with primary antibodies against ZEB1 (ab181451; 1:1,000 dilution, Abcam, Cambridge, UK) or GAPDH (ab110305; 1:1,000 dilution, Abcam). On the following day, the membranes were washed with TBST and probed with goat anti-mouse horseradish-peroxidase-conjugated secondary antibody (ab6789; 1:5,000 dilution, Abcam) at room temperature for $2 \mathrm{~h}$. The protein signals were visualized using Pierce ${ }^{\mathrm{TM}}$ ECL Western Blotting Substrate (Pierce; Thermo Fisher Scientific, Inc.). GAPDH was used as an internal control. Quantity One software (version 4.62; Bio-Rad Laboratories, Inc.) was used for densitometry.

Statistical analysis. Each assay was repeated at least three times. All data were analyzed using SPPS version 19.0 (IBM Corp., Armonk, NY, USA). The expression of miR-873 and ZEB1 mRNA in TC was presented as box plots, and a Mann-Whitney U test was used to analyze these data. The
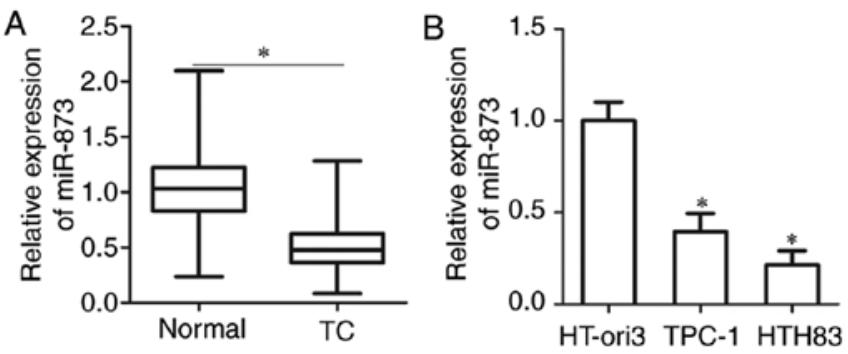

Figure 1. miR-873 is downregulated in TC tissues and cell lines. (A) Relative expression of miR-873 was analysed in 35 pairs of TC and adjacent normal tissues using RT-qPCR ( $\mathrm{n}=35)$. ${ }^{*} \mathrm{P}<0.05$. (B) miR-873 expression was determined in one normal human thyroid cell line (HT-ori3) and two human TC cell lines (TPC-1 and HTH83) using RT-qPCR. " $\mathrm{P}<0.05$ vs. HT-ori3. miR, microRNA; TC, thyroid cancer; RT-qPCR, reverse transcription-quantitative polymerase chain reaction.

top of the box indicated the upper quartile, while the bottom presented the lower quartile. The central line in the box indicated the median. In addition, the whiskers indicated the minimum and maximum data. All other data were expressed as the mean \pm standard deviation, and differences between groups were evaluated using a two-tailed Student's t-test or one-way ANOVA. A Tukey's test was applied as the post hoc test following ANOVA. Spearman's correlation analysis was conducted to determine the association between miR-873 and ZEB1 mRNA expression in TC tissues. $\mathrm{P}<0.05$ was considered to indicate a statistically significant difference.

\section{Results}

miR-873 is downregulated in human TC tissues and cell lines. RT-qPCR analysis was performed to detect miR-873 expression in 35 pairs of TC and adjacent normal tissues. The results of RT-qPCR analysis showed that miR-873 was downregulated in TC tissues compared with in adjacent normal tissues $(\mathrm{P}<0.05$; Fig. 1A). To further validate this observation, the expression of miR-873 was investigated in a normal human thyroid cell line (HT-ori3) and two human TC cell lines (TPC-1, and HTH83). Consistently, the expression levels of miR-873 were significantly decreased in the TC cell lines than in HT-ori3 cells $(\mathrm{P}<0.05$; Fig. 1B). These findings suggested that miR-873 was downregulated in TC tissues and cell lines.

miR-873 inhibits the proliferation and invasion of TC cells. To determine the roles of miR-873 in TC, TPC- 1 and HTH83 cells were transfected with miR-873 mimics or miR-NC. miR-873 expression was significantly upregulated in TPC-1 and HTH 83 cells following transfection with miR-873 mimics, as confirmed by RT-qPCR analysis $(\mathrm{P}<0.05$; Fig. $2 \mathrm{~A})$. To examine the effects of miR-873 on cellular proliferation, MTT assays were conducted to detect the proliferation of TPC-1 and HTH83 cells after overexpressing miR-873. Cell proliferation was significantly reduced in miR-873 mimics-transfected TPC- 1 and HTH 83 cells at 48 and $72 \mathrm{~h}$ relative to that of cells transfected with miR-NC $(\mathrm{P}<0.05$; Fig. 2B). Additionally, the invasive abilities of TPC-1 and HTH83 cells transfected with miR-873 mimics or miR-NC were determined via a cell invasion assay. The results demonstrated that miR-873 overexpression significantly reduced the invasion of TPC-1 

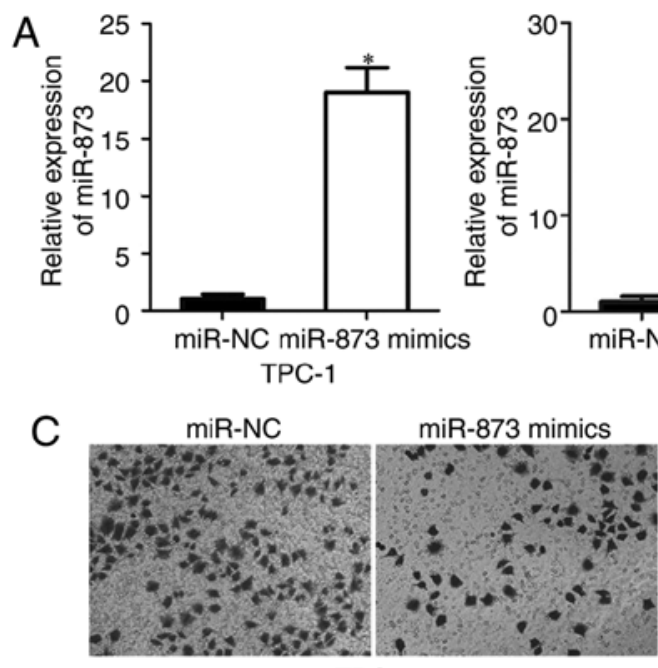

TPC-1
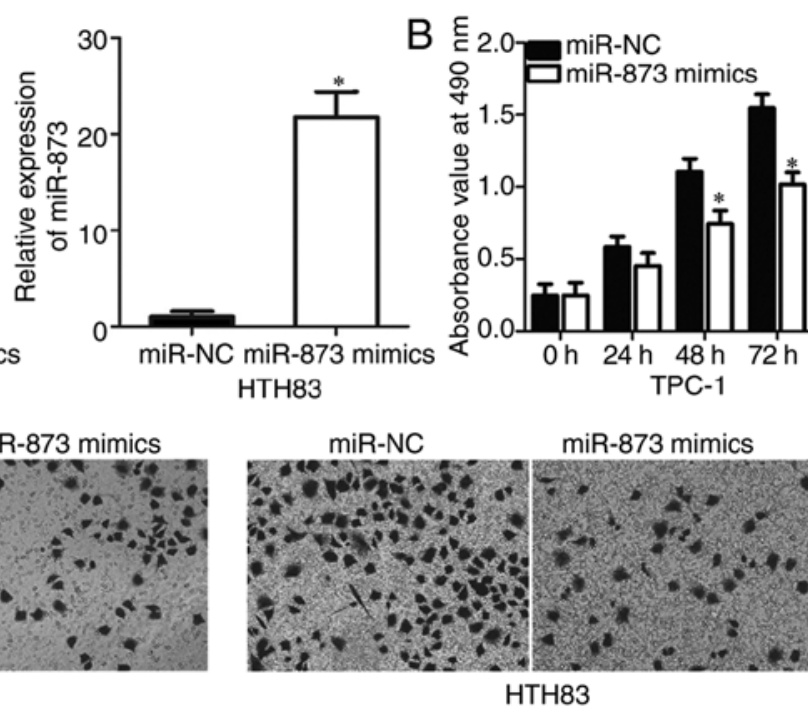
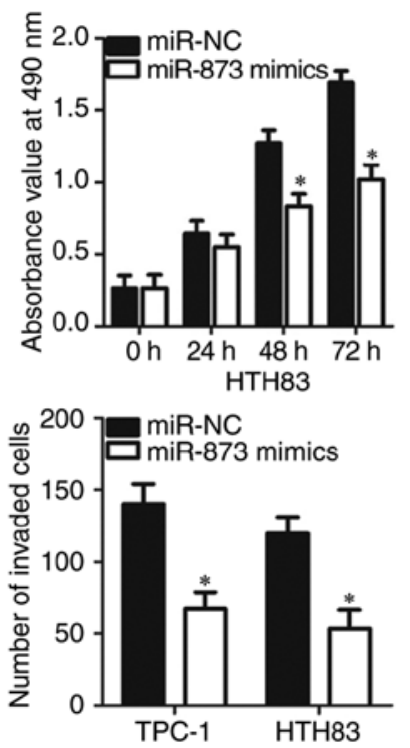

Figure 2. miR-873 overexpression restricts the proliferation and invasion of TPC-1 and HTH83 cells. miR- 873 mimics or miR-NC was transfected into TPC-1 and HTH83 cells. (A) At $48 \mathrm{~h}$ post-transfection, transfected cells were subjected to RT-qPCR analysis for the detection of miR- 873 expression. ${ }^{*} \mathrm{P}<0.05$ vs. miR-NC. (B) An MTT assay was performed to measure cell proliferation at various time points. ${ }^{*} \mathrm{P}<0.05$ vs. miR-NC. (C) At $48 \mathrm{~h}$ post-transfection, cell invasion was evaluated with a cell invasion assay (magnification, $\mathrm{x} 200$ ). ${ }^{*} \mathrm{P}<0.05$ vs. miR-NC. miR, microRNA; NC, negative control.

and HTH83 cells compared with the control group $(\mathrm{P}<0.05$; Fig. 2C). Collectively, the results indicated that miR-873 may serve inhibitory roles in the progression of TC.

miR-873 decreases ZEB1 expression in TC cells by binding to its 3'-UTR. To understand the mechanisms by which miR-873 inhibited TC cell proliferation and invasion in the present study, bioinformatics analysis was conducted to predict the candidate targets of miR-873. ZEB1 was predicted as a major putative target of miR-873 (Fig. 3A) and was closely associated with the formation and progression of TC (23). Therefore, ZEB1 was selected for further experimental confirmation. To clarify whether miR-873 could bind to the 3'-UTR of ZEB1, luciferase reporter plasmids were constructed and transfected into TPC-1 and HTH83 cells, together with miR-873 mimics or miR-NC. The results of the luciferase reporter assay indicated that upregulation of miR-873 significantly decreased the luciferase activities of the plasmid carrying Wt ZEB1 3'-UTR in TPC-1 and HTH83 cells compared with the corresponding miR-NC group $(\mathrm{P}<0.05)$; however, the luciferase activity of the plasmid harbouring Mut ZEB1 3'-UTR was markedly unaffected (Fig. 3B). To further investigate the regulatory effects of miR-873 on endogenous ZEB1 expression in TC cells, TPC-1 and HTH83 cells were transfected with miR-873 mimics or miR-NC. The expression of ZEB1 mRNA and protein levels were then determined. RT-qPCR and western blot analysis showed that miR-873 upregulation significantly suppressed ZEB1 expression in TPC-1 and HTH83 cells at the mRNA $(\mathrm{P}<0.05$; Fig. $3 \mathrm{C})$ and protein $(\mathrm{P}<0.05$; Fig. 3D) levels compared with the control. Collectively, these results suggested that ZEB1 may be a direct target gene of miR-873 in TC cells.

ZEB1 exhibits an inverse correlation with $\mathrm{miR}-873$ expression in TC tissues. To further investigate the correlation between miR-873 and ZEB1 in TC, we detected ZEB1 expression in 35 pairs of $\mathrm{TC}$ and adjacent normal tissues. Our results showed that the expression of ZEB1 was upregulated in TC tissues compared with in adjacent normal tissues at the mRNA ( $\mathrm{P}<0.05$; Fig. 4A) and protein levels (Fig. 4B). Then, the correlation between miR-873 and ZEB1 mRNA levels was evaluated using Spearman's correlation analysis. An inverse correlation between miR-873 and ZEB1 mRNA levels was observed in TC tissues ( $r=-0.5275, P=0.00111$; Fig. 4C).

Restoration of ZEB1 expression rescues the inhibitory effects of miR-873 on cell proliferation and invasion in TC. To investigate whether the inhibitory effects of miR-873 on TC cell proliferation and invasion were mediated by ZEB1, we restored ZEB1 expression in miR-873-overexpressing TPC-1 and HTH83 cells via co-transfection with ZEB1 overexpression plasmid pcDNA3.1-ZEB1 ( $\mathrm{P}<0.05$; Fig. 5A). Functional assays revealed that restoration of ZEB1 expression could rescue the suppressive effects of miR-873 overexpression on the proliferation $(\mathrm{P}<0.05$; Fig. $5 \mathrm{~B})$ and invasion $(\mathrm{P}<0.05$; Fig. 5C) of TPC-1 and HTH83 cells. These results suggested that miR-873 may exert its tumour-suppressing roles in TC at least partly by suppressing ZEB1.

\section{Discussion}

Previous studies have reported that certain miRNAs are dysregulated in TC, which is linked with the pathogenesis and development of TC (24-26). Therefore, improved understanding of the detailed roles of miRNAs that are aberrantly expressed in TC may aid the development of valuable therapeutic methods for the management of patients with this malignancy. The present study demonstrated that miR-873 expression was reduced in both TC tissues and cell lines. Ectopic miR-873 expression inhibited cell proliferation and invasion in TC. In addition, ZEB1 was identified as a direct target gene of miR-873 in TC. Furthermore, ZEB1 was 

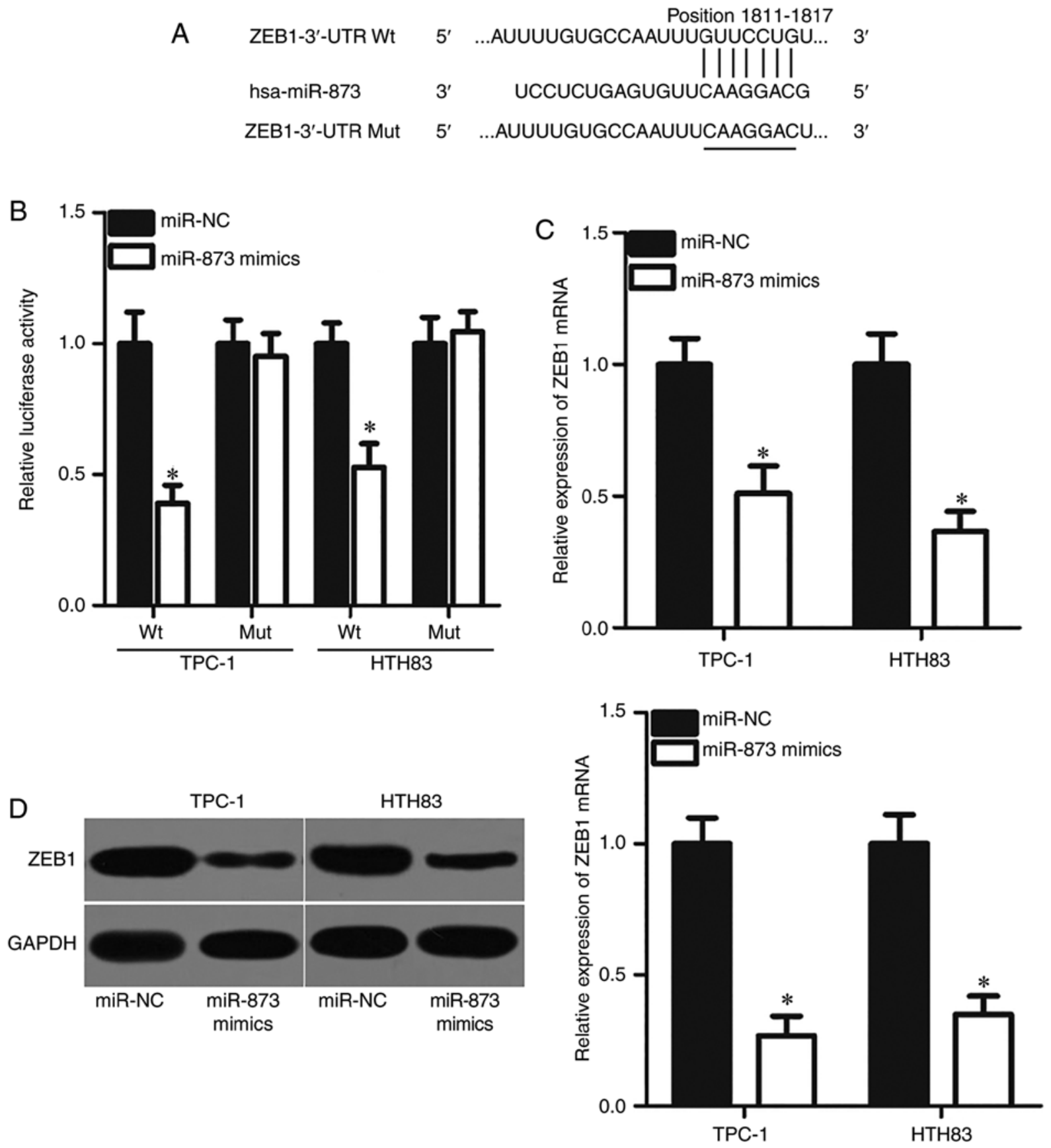

Figure 3. miR-873 directly targets ZEB1 in TPC-1 and HTH83 cells. (A) Predicted Wt binding sites for miR-873 within the 3'-UTR of ZEB1. The mutant binding sequences were also presented. (B) Luciferase reporter plasmids containing the Wt or Mut miR-873 binding sequences in the 3'-UTR of ZEB1 were constructed and transfected into TPC-1 and HTH83 cells along with miR-873 mimics or miR-NC. Luciferase activities were detected at $48 \mathrm{~h}$ post-transfection. ${ }^{*} \mathrm{P}<0.05$ vs. miR-NC. The levels of ZEB1 (C) mRNA and (D) protein were determined in TPC-1 and HTH83 cells transfected with miR-873 mimics or miR-NC using reverse transcription-quantitative polymerase chain reaction and western blot analysis, respectively. ${ }^{*} \mathrm{P}<0.05$ vs. miR-NC. Hsa, homo sapiens; miR, microRNA; Mut, mutant; NC, negative control; Wt, wild-type; ZEB1, zinc finger E-box-binding homeobox 1.

upregulated in TC tissues, and negatively correlated with the expression of miR-873. Additionally, the resumption of ZEB1 expression rescued TC cells from the tumour-suppressive effects of miR-873 on cell proliferation and invasion. These results suggested that miR-873 may serve as a potential therapeutic target for treating patients with TC.

miR-873 was reported to be aberrantly expressed in several types of human cancer. For example, this miRNA was downregulated in colorectal cancer tissues and cell lines (17). Decreased miR-873 expression has been associated with the tumour stage of patients with colorectal cancer. In addition, patients with this disease and low miR-873 expression levels exhibited reduced overall survival rates than those with high expression levels (17). miR-873 was determined to be downregulated in gastric cancer tissues and cell lines $(19,20)$. Reduced miR-873 expression strongly correlated with the levels of serum $\alpha$ fetal protein, tumour size and, tumour-node-metastasis stage of patients with gastric cancer (20). miR-873 downregulation was also reported in breast cancer (18), glioblastoma (27) and ovarian cancer (28); however, miR-873 was upregulated 

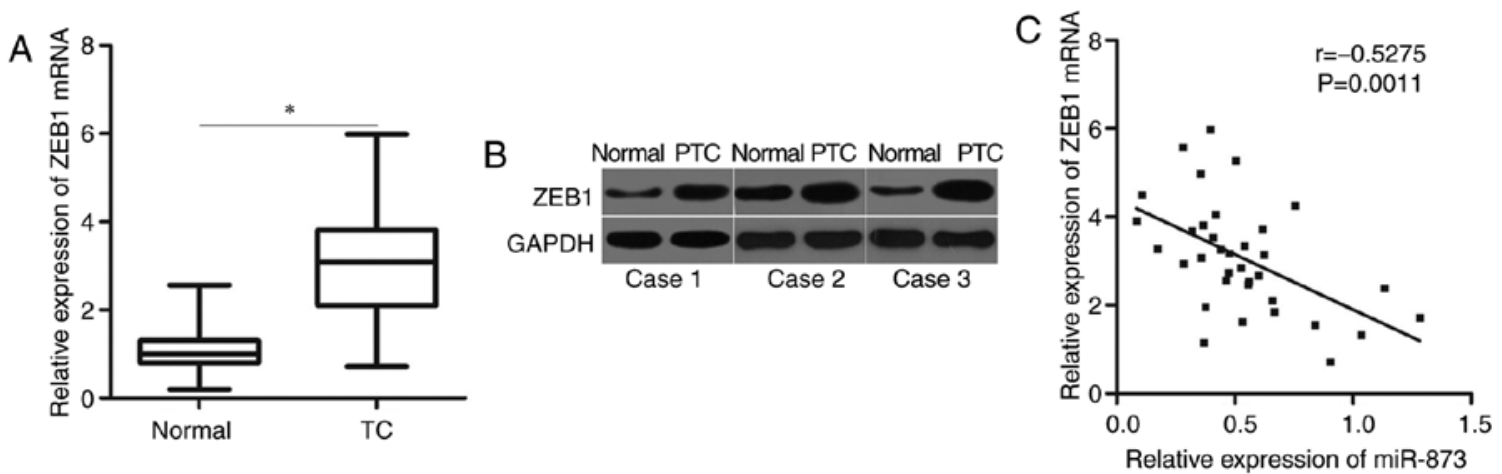

Figure 4. ZEB1 is overexpressed in TC tissues, and its overexpression is negatively correlated with miR-873 expression. ZEB1 (A) mRNA and (B) protein expression in TC and adjacent normal tissues was determined by reverse transcription-quantitative polymerase chain reaction $(\mathrm{n}=35)$ and western blot analysis $(\mathrm{n}=3)$, respectively. ${ }^{*} \mathrm{P}<0.05$ vs. adjacent normal tissues. (C) Spearman's correlation analysis of the association between miR-873 and ZEB1 mRNA expression levels in TC tissues. $\mathrm{r}=-0.5275, \mathrm{P}=0.0011$. TC, thyroid cancer; miR, microRNA; ZEB1, zinc finger E-box-binding homeobox 1.

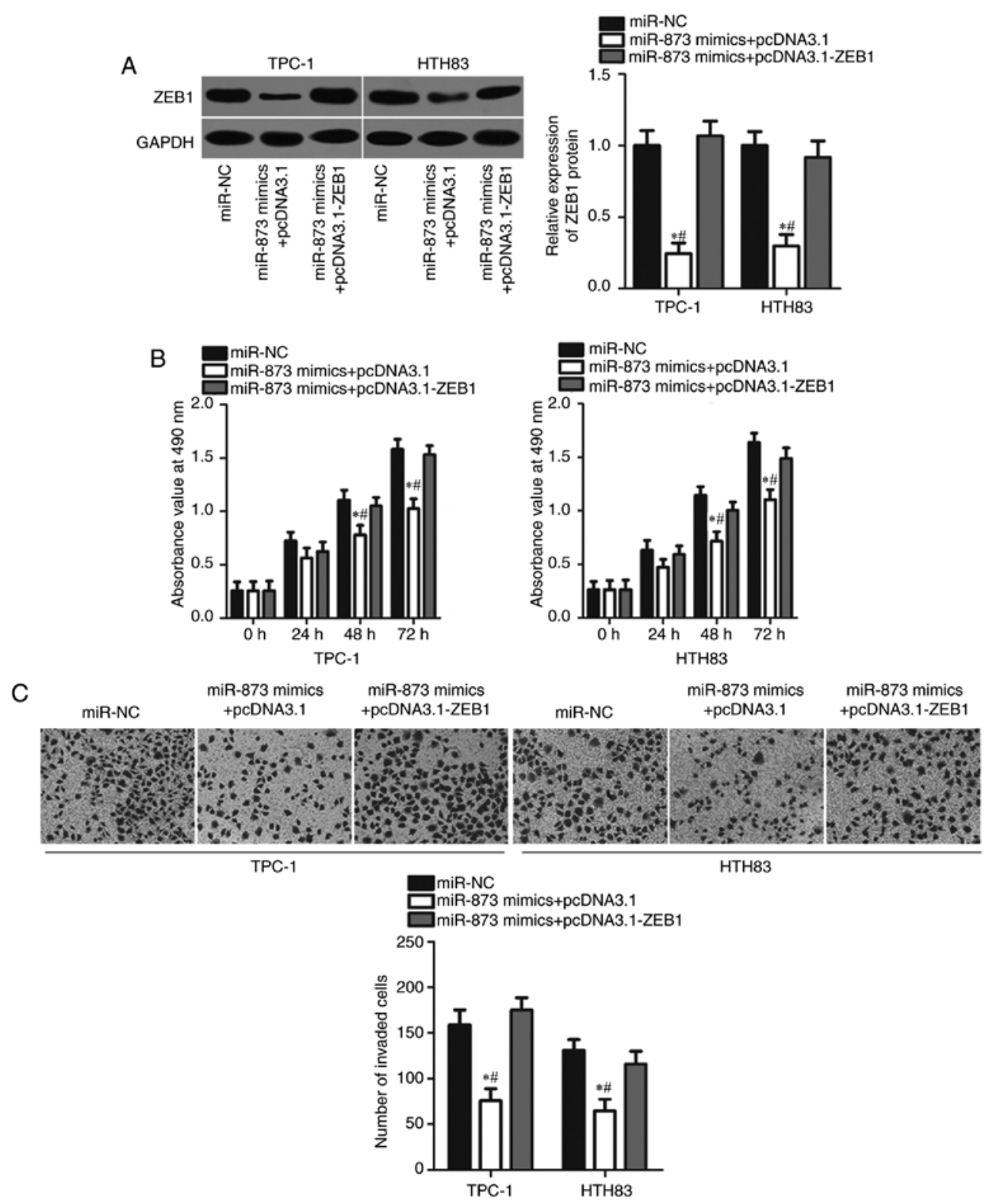

Figure 5. Ectopic expression of ZEB1 rescues the effects of miR-873 overexpression on TPC-1 and HTH83 cell proliferation and invasion. TPC-1 and HTH83 cells were co-transfected with miR-873 mimics and pcDNA3.1-ZEB1 or empty plasmid pcDNA3.1. (A) At $72 \mathrm{~h}$ post-transfection, ZEB1 protein expression was measured using western blot analysis. ${ }^{*} \mathrm{P}<0.05$ vs. miR-NC. ${ }^{~} \mathrm{P}<0.05$ vs. miR-873 mimics + pcDNA3.1-ZEB1. MTT and cell invasion assays (magnification, $\mathrm{x} 200$ ) were carried out to assess (B) cell proliferation and (C) invasion in indicated cells. ${ }^{*} \mathrm{P}<0.05$ vs. miR-NC; ${ }^{"} \mathrm{P}<0.05$ vs. miR-873 mimics + pcDNA3.1-ZEB1. miR-NC. miR, microRNA; NC, negative control; ZEB1, zinc finger E-box-binding homeobox 1. 
in lung adenocarcinoma tissues and cell lines (29). These conflicting findings indicate the potential tissue specificity of miR-873 expression in human malignancies, and that miR-873 may serve as a biomarker for the diagnosis and prognosis of these human specific cancer types.

miR-873 exhibits tumour-suppressive effects in numerous types of human cancer. For instance, miR-873 suppresses the proliferation of colorectal cancer cells in vitro (17). Cui et al (18) reported that miR-873 overexpression inhibits breast cancer cell proliferation in vitro and tumour growth in vivo. Cao et al (19) and Chen et al (20) revealed that resumption of miR-873 expression suppresses cell viability, promotes cell apoptosis and induces cell cycle arrest in gastric cancer. Wang et al (27) revealed that miR-873 overexpression attenuates cell growth and metastasis of glioblastoma in vitro. Additionally, Wu et al (28) found that miR-873 overexpression increases the chemosensitivity of ovarian cancer cells to cisplatin and paclitaxel by enhancing the inhibitory effects of these drugs on tumour growth. Nevertheless, miR-873 serves oncogenic roles in lung adenocarcinoma by affecting cell proliferation and migration in vitro (29). These findings demonstrated that miR-873 exhibits tissue-specific effects on the biological roles involved in tumourigenesis. These contradictory results may be explained by the imperfect complementarity of the interactions between miRNAs and target genes (30). These findings also suggested that miR-873 may be considered as a potential therapeutic target for the management of these specific types of tumour.

Direct targets of miR-873 have been previously validated, including tumour necrosis factor receptor-associated factor 5 (17) and transforming growth factor- $\beta$ activated kinase 1 (17) in colorectal cancer, cyclin-dependent kinase 3 (18) in breast cancer, glioma-associated oncogene (19) and C-X-C motif ligand 1 (20) in gastric cancer, insulin-like growth factor 2 mRNA-binding protein 1 in glioblastoma (27), ATP-binding cassette sub-family B member 1 (28) in ovarian cancer and SRC kinase signaling inhibitor 1 (29) in lung cancer. ZEB1, located on the short arm of human chromosome 10 (31), has been reported as a direct and functional target of miR-873 in TC. It is a member of the zinc finger family and is highly expressed in various human cancers, such as cervical cancer (32), lung cancer (33), hepatocellular carcinoma (34), gastric cancer (35) and glioma (36). ZEB1 is also upregulated in TC and was strongly associated with tumour-node-metastasis stage, lymph node metastasis and distant metastasis (23). ZEB1 inhibition suppressed the proliferation, migration and invasion of TC cells (23). In the present study, we revealed that miR-873 may inhibit TC progression by downregulating ZEB1 expression. Thus, the inhibition of ZEB1 via miR-873 may be an effective therapeutic strategy to treat patients with TC.

Overall, miR-873 was downregulated in TC tissues and cell lines. In addition, overexpression of miR-873 may have attenuated TC cell proliferation and invasion by directly targeting ZEB1. Our experimental results suggested that this miRNA may be a promising therapeutic target for the treatment of TC patients; however, the association between miR-873, ZEB1 and the clinical characteristics of patients with $\mathrm{TC}$ requires further investigation. In addition, experiments, including colony formation and wound healing assays, and in vivo xenograft experiments should be performed to explore the roles of
miR-873 in the progression of TC. These are limitations of our study, which we aim to resolve in future.

\section{Acknowledgements}

Not applicable.

\section{Funding}

No funding was received.

\section{Availability of data and materials}

The datasets used and/or analyzed during the present study are available from the corresponding author on reasonable request.

\section{Authors' contributions}

QF and DJ made substantial contributions to the design of this research. DJ, FG and QF performed the functional assays. All authors read and approved the final draft.

\section{Ethics approval and consent to participate}

The present study was approved by the Ethics Committee of China-Japan Union Hospital of Jilin University, and was performed in accordance with the Declaration of Helsinki and the guidelines of the Ethics Committee of China-Japan Union Hospital of Jilin University. Written informed consent was obtained from all patients for the use of their clinical tissues.

\section{Patient consent for publication}

Not applicable.

\section{Competing interests}

The authors declare that they have no competing interests.

\section{References}

1. Liebner DA and Shah MH: Thyroid cancer: Pathogenesis and targeted therapy. Ther Adv Endocrinol Metab 2: 173-195, 2011.

2. Lloyd RV, Buehler D and Khanafshar E: Papillary thyroid carcinoma variants. Head Neck Pathol 5: 51-56, 2011.

3. Das S, Chaudhary N, Ang LC and Megyesi JS: Papillary thyroid carcinoma metastasizing to anaplastic meningioma: An unusual case of tumor-to-tumor metastasis. Brain Tumor Pathol 34: 130-134, 2017.

4. Nikiforov YE and Nikiforova MN: Molecular genetics and diagnosis of thyroid cancer. Nat Rev Endocrinol 7: 569-580, 2011.

5. Liu S, Semenciw R, Ugnat AM and Mao Y: Increasing thyroid cancer incidence in Canada, 1970-1996: Time trends and age-period-cohort effects. Br J Cancer 85: 1335-1339, 2001.

6. Pemayun TG: Current diagnosis and management of thyroid nodules. Acta Med Indones 48: 247-257, 2016.

7. Lu J, Getz G, Miska EA, Alvarez-Saavedra E, Lamb J, Peck D, Sweet-Cordero A, Ebert BL, Mak RH, Ferrando AA, et al: MicroRNA expression profiles classify human cancers. Nature 435: 834-838, 2005 .

8. Jia L, Zhang D, Qi X, Ma B, Xiang Z and He N: Identification of the conserved and novel miRNAs in Mulberry by high-throughput sequencing. PLoS One 9: e104409, 2014.

9. Xie B,Ding Q,Han Hand Wu D: miRCancer: A microRNA-cancer association database constructed by text mining on literature. Bioinformatics 29: 638-644, 2013. 
10. Mutalib NS, Yusof AM, Mokhtar NM, Harun R, Muhammad R and Jamal R: MicroRNAs and lymph node metastasis in papillary thyroid cancers. Asian Pac J Cancer Prev 17: 25-35, 2016.

11. Xu J, Li J, Zheng TH, Bai L and Liu ZJ: MicroRNAs in the occurrence and development of primary hepatocellular carcinoma Adv Clin Exp Med 25: 971-975, 2016.

12. Hamam R, Hamam D, Alsaleh KA, Kassem M, Zaher W, Alfayez M, Aldahmash A and Alajez NM: Circulating microRNAs in breast cancer: Novel diagnostic and prognostic biomarkers. Cell Death Dis 8: e3045, 2017.

13. Irmak-Yazicioglu MB: Mechanisms of MicroRNA deregulation and MicroRNA targets in gastric cancer. Oncol Res Treat 39: 136-139, 2016.

14. Zhang M, Wu W, Gao M and Fei Z: MicroRNA-451 as a prognostic marker for diagnosis and lymph node metastasis of papillary thyroid carcinoma. Cancer Biomark 19: 437-445, 2017.

15. Liu J, Li Q, Li R, Ren P and Dong S: MicroRNA-363-3p inhibits papillary thyroid carcinoma progression by targeting PIK3CA Am J Cancer Res 7: 148-158, 2017.

16. Zhao H, Tang H, Huang Q, Qiu B, Liu X, Fan D, Gong L, Guo H, Chen C, Lei S, et al: MiR-101 targets USP22 to inhibit the tumorigenesis of papillary thyroid carcinoma. Am J Cancer Res 6: 2575-2586, 2016.

17. Gong H, Fang L, Li Y, Du J, Zhou B, Wang X, Zhou H, Gao L, Wang $K$ and Zhang J: miR873 inhibits colorectal cancer cell proliferation by targeting TRAF5 and TAB1. Oncol Rep 39: 1090-1098, 2018.

18. Cui J, Yang Y, Li H, Leng Y, Qian K, Huang Q, Zhang C, Lu Z, Chen J, Sun T, et al: MiR-873 regulates ER $\alpha$ transcriptional activity and tamoxifen resistance via targeting CDK3 in breast cancer cells. Oncogene 34: 4018, 2015.

19. Cao D, Yu T and Ou X: MiR-873-5P controls gastric cancer progression by targeting hedgehog-GLI signaling. Pharmazie 71: 603-606, 2016

20. Chen XY, Chen RP, Wu W and Huang ZM: MicroRNA-873 inhibits proliferation and induces apoptosis by targeting CXCL1 in gastric cancer. Int J Clin Exp Pathol 9: 10011-10019, 2016.

21. Liu ZY, Zhou GY, Kakudo K and Lam AK: Update on 2017 Who classification of tumors of thyroid gland. Zhonghua Bing Li Xue Za Zhi 47: 302-306, 2018 (In Chinese).

22. Livak KJ and Schmittgen TD: Analysis of relative gene expression data using real-time quantitative PCR and the 2(-Delta Delta C(T)) method. Methods 25: 402-408, 2001.

23. Zhang Y, Liu G, Wu S, Jiang F, Xie J and Wang Y: Zinc finger E-box-binding homeobox 1: Its clinical significance and functional role in human thyroid cancer. Onco Targets Ther 9: 1303-1310, 2016.
24. Wang Z, Zhang H, He L, Dong W, Li J, Shan Z and Teng W: Association between the expression of four upregulated miRNAs and extrathyroidal invasion in papillary thyroid carcinoma. Onco Targets Ther 6: 281-287, 2013.

25. Graham ME, Hart RD, Douglas S, Makki FM, Pinto D, Butler AL, Bullock M, Rigby MH, Trites JR, Taylor SM and Singh R: Serum microRNA profiling to distinguish papillary thyroid cancer from benign thyroid masses. J Otolaryngol Head Neck Surg 44: 33, 2015.

26. Lee JC, Zhao JT, Clifton-Bligh RJ, Gill A, Gundara JS, Ip JC, Glover A, Sywak MS, Delbridge LW, Robinson BG and Sidhu SB: MicroRNA-222 and microRNA-146b are tissue and circulating biomarkers of recurrent papillary thyroid cancer. Cancer 119: 4358-4365, 2013

27. Wang RJ, Li JW, Bao BH, Wu HC, Du ZH, Su JL, Zhang MH and Liang HQ: MicroRNA-873 (miRNA-873) inhibits glioblastoma tumorigenesis and metastasis by suppressing the expression of IGF2BP1. J Biol Chem 290: 8938-8948, 2015

28. Wu DD, Li XS, Meng XN, Yan J and Zong ZH: MicroRNA-873 mediates multidrug resistance in ovarian cancer cells by targeting ABCB1. Tumour Biol 37: 10499-10506, 2016.

29. Gao Y, Xue Q, Wang D, Du M, Zhang Y and Gao S: miR-873 induces lung adenocarcinoma cell proliferation and migration by targeting SRCIN1. Am J Transl Res 7: 2519-2526, 2015.

30. Yu Z, Ni L, Chen D, Zhang Q, Su Z, Wang Y, Yu W, Wu X, Ye J, Yang S, et al: Identification of miR-7 as an oncogene in renal cell carcinoma. J Mol Histol 44: 669-677, 2013.

31. Zhang Y, Xu L, Li A and Han X: The roles of ZEB1 in tumorigenic progression and epigenetic modifications. Biomed Pharmacother 110: 400-408, 2019.

32. Ma Y, Zheng X, Zhou J, Zhang Y and Chen K: ZEB1 promotes the progression and metastasis of cervical squamous cell carcinoma via the promotion of epithelial-mesenchymal transition. Int J Clin Exp Pathol 8: 11258-11267, 2015.

33. Larsen JE, Nathan V, Osborne JK, Farrow RK, Deb D, Sullivan JP, Dospoy PD, Augustyn A, Hight SK, Sato M, et al: ZEB1 drives epithelial-to-mesenchymal transition in lung cancer. J Clin Invest 126: 3219-3235, 2016.

34. Zhou YM, Cao L, Li B, Zhang RX, Sui CJ, Yin ZF and Yang JM: Clinicopathological significance of ZEB1 protein in patients with hepatocellular carcinoma. Ann Surg Oncol 19: 1700-1706, 2012.

35. Jia B, Liu H, Kong Q and Li B: Overexpression of ZEB1 associated with metastasis and invasion in patients with gastric carcinoma. Mol Cell Biochem 366: 223-229, 2012.

36. Guo E, Wang Z and Wang S: MiR-200c and miR-141 inhibit ZEB1 synergistically and suppress glioma cell growth and migration. Eur Rev Med Pharmacol Sci 20: 3385-3391, 2016. 